\title{
Evaluation of the prognostic value of the risk, injury, failure, loss and end-stage renal failure (RIFLE) criteria for acute kidney injury
}

\author{
Jose R. Perez Valdivieso, ${ }^{1}$ Maira Bes-Rastrollo, ${ }^{2}$ Pablo Monedero, ${ }^{1}$ Jokin De Irala ${ }^{2}$ and \\ Francisco J. Lavilla ${ }^{3}$ \\ ${ }^{1}$ Department of Anaesthesia and Critical Care, ${ }^{2}$ Department of Preventive Medicine and \\ Public Health, and ${ }^{3}$ Renal Unit, University Hospital of Navarra, University of Navarra, \\ Pamplona (Navarra), Spain
}

\section{SUMMARY}

Aim: The experts have argued about the use of the risk, injury, failure, loss and endstage renal failure (RIFLE) criteria as a prognosis scoring system. We examined the association between in-hospital mortality and the RIFLE criteria, and discussed its accuracy as a prognosis factor.

Methods: In this prospective study, we analysed the data gathered from a cohort of 956 patients admitted in a Spanish tertiary hospital between January 1998 and April 2006. Hazard ratios for mortality, and survival curves within 60 days were calculated. Discrimination and calibration of the model were also assessed.

Results: Excluding 53 patients, 903 patients were finally analysed. We classified them into groups according to the maximum RIFLE class reached during their admission. The RIFLE class was assessed by the glomerular filtration rate criterion. We found an increase in the in-hospital mortality risk. Cox proportional hazard models showed that RIFLE classes risk, injury, and failure were significant predictive factors (hazard ratios were $2.77,3.23$ and 3.52, respectively; $\mathrm{P}$ for trend was 0.005 ). The multivariate analyses from the cross-classification of the participants according to Liano score values (severity of illness) and RIFLE classes showed additive effects of the exposures on inhospital mortality.

Conclusion: In this population, the risk of in-hospital mortality during the acute kidney injury (AKI) episode was positively associated with RIFLE classes. We showed that the RIFLE classification system had discriminative power in predicting hospital mortality within 60 days in AKI patients, but not better than a specific AKI predictive model. However, a combined use of both may give a more robust prognosis system.

Key words: acute kidney injury, Liano score, mortality, prognosis, RIFLE criteria.

Correspondence: Dr Jose Ramon Perez Valdivieso

Department of Anaesthesia and Critical Care, Clinica Universitaria, Pio XII, 36. 31008-Pamplona, Spain.

Email: jrpvaldi@unav.es 


\section{INTRODUCTION}

Acute kidney injury (AKI) is an increasingly common syndrome. ${ }^{1-3}$ Although a decrease in mortality has been reported using administrative databases, ${ }^{1,2}$ the lack of detailed clinical and laboratory information, and the existence of changing coding practices limits the interpretation. ${ }^{3}$ Moreover, clinical reports and reviews continue reporting high mortality rates, ${ }^{4-8}$ despite advances in medical care. These mortality rates vary widely based on the type of clinical setting, and due to the differences of AKI definitions.

More than 30 different definitions of AKI have been published to date, ${ }^{9}$ thus making both research and clinical work difficult. The Acute Dialysis Quality Initiative (ADQI) developed a consensus definition of AKI: the risk, injury, failure, loss and end-stage renal failure (RIFLE) criteria. ${ }^{9}$ The RIFLE criteria provides a graded classification of AKI severity (risk, injury and failure), corresponding the final two stages to clinical outcomes. This unified definition could prove useful in conducting better-designed clinical research in the form of patient stratification, centre-to-centre comparisons, as well as assessing new interventions. ${ }^{10}$

The use of RIFLE as a classification system warrants worldwide clinical validation before applying the criteria to a clinical setting. Furthermore, such an approach is specifically more relevant when there are apparent controversies surrounding this issue. On one hand, Maccariello et al. ${ }^{11}$ evaluated the RIFLE classification and outcomes in a cohort of critically ill patients, and Joannidis gave his support to it in an associated editorial. ${ }^{12}$ On the other hand, Bellomo et al. ${ }^{13}$ and Kellum et al. ${ }^{14}$ have expressed their concerns about the misuse and misinterpretation of the RIFLE criteria that, in their opinion, the previous articles showed. They recommended that the RIFLE classification should not be used as a prognostic scoring system, and that the RIFLE classification should not be used at a single moment in time, as Maccariello did.

Because we consider these topics of interest, the objective of this study was to evaluate what association may exist between the RIFLE criteria and in-hospital mortality, as well as to examine the predictive capacity of the RIFLE criteria. We think this work can shed some light on these current issues.

\section{METHODS}

\section{Study population}

We conducted this prospective observational study between January 1998 and April 2006 at the University Hospital of Navarra, in the city of Pamplona, Spain. This is a 400-bed tertiary care teaching centre. During this period, 988 cases in 956 admitted patients who had had a nephrology consultation requested, because of suspicion of AKI, were prospectively entered into a computerized database. We excluded patients who: (i) presented with oliguria but did not show an adequate serum creatinine increase to qualify for one of the creatinine RIFLE criteria $(n=44)$; (ii) were younger than 16 years $(\mathrm{n}=1)$; or (iii) had missing data $(\mathrm{n}=8)$. In the event of multiple admissions (32 cases), only the initial admission was considered to avoid bias. Therefore, 903 patients remained for further analysis. The hospital ethics committee approved the study. 


\section{Data collection and definitions}

The following demographic and clinical data were collected at the time of nephrology consultation: age, gender, current Liano score and the elements of the equation, ${ }^{15,16}$ Karnofsky score ${ }^{17}$ at home, admission type (surgical or medical), presence of oncology disease or not, previous history of chronic renal failure, occurrence of diabetes or not, aetiology of AKI (pre-renal, renal, post-renal, and other causes), and prior food intake. In addition, information such as subsequent treatment, or further examination of serum creatinine levels were ascertained. Finally, whether the patients died within 60 days after the start of the nephrology consultation was also noted.

To obtain the Liano score, the following equation was used: ${ }^{15} 0.032 \mathrm{x}$ age in decades $0.086 \mathrm{x}$ male gender $-0.109 \mathrm{x}$ nephrotoxic $+0.109 \mathrm{x}$ oliguria $+0.116 \mathrm{x}$ hypotension + $0.122 \mathrm{x}$ jaundice $+0.150 \mathrm{x}$ coma $-0.154 \mathrm{x}$ consciousness $+0.182 \mathrm{x}$ assisted respiration +0.210 .

We classified all the in-patients according to the highest RIFLE category during the hospital admission (i.e. risk, injury and failure). ${ }^{9}$ Those patients who presented with neither oliguria nor adequate increase of serum creatinine were included in the 'no AKI' group. However, we did not study the outcome classes of RIFLE (i.e. loss and end-stage kidney disease).

We calculated the baseline creatinine in patients with no previous history of chronic renal disease using the modification of diet in renal disease (MDRD) equation, ${ }^{18}$ as recommended by the ADQI, ${ }^{9}$ assuming a glomerular filtration rate of $75 \mathrm{~mL} / \mathrm{min}$ per $1.73 \mathrm{~m}^{2}$. For patients with a known history of chronic renal disease, the baseline serum creatinine was assumed to be the one that was measured at the time of hospital admission.

Food intake was defined as the caloric ingestion in the previous days, and this was classified as 'appropriate' when it was optimal, 'mild malnutrition' when it had been inadequate for less than 3 days, 'moderate malnutrition' when it had been inadequate between 3 and 7 days, and 'severe malnutrition' when it had been inadequate for more than 7 days.

All cases were treated during the admission by the same nephrologist, and all the data were gathered by the same observer.

The primary outcome variable was in-hospital mortality within 60 days.

\section{Statistical analysis}

Continuous data were presented as medians (and interquartile ranges), and compared via ANovA. Categorical variables were expressed as proportions and compared using Fisher's exact test.

Cox proportional hazards models were fitted to assess the relationship between RIFLE categories (no evidence of AKI, risk (R), injury (I) and failure (F)) and the risk of inhospital mortality. The time of origin was the date when the nephrology consultation started. The event defined was death, whereas those cases alive at the end of follow up and those lost to follow up were censored at their last observation. Hazard ratios (HR) and their 95\% confidence intervals (CI) were calculated considering the group with no AKI as the reference category. We fitted a crude model (univariate; i.e. without any adjustment), an age and gender model, and a multivariate adjusted model including the following variables selected by the descriptive analysis of potential confounders: Liano score, prior food intake, need for renal replacement therapy, chronic renal failure, the cause of AKI, admission type (surgical or not) and Karnofsky score. We additionally adjusted for oncologic disease because of a higher proportion of oncology patients in 
our cohort $(37 \%)$. We calculated $\mathrm{P}$ for trend considering RIFLE categories as a continuous variable.

We analysed overall survival at 60 days across groups using the Kaplan-Meier methods, and tested differences between groups using the log-rank test.

Calibration of the model was assessed by the goodness-of-fit statistic test from HosmerLemeshow, and graphically displayed by plotting observed and predicted mortality for all patients across all risk ranges. Discrimination power was evaluated by determining the area under the receiver-operating curve (ROC).

We conducted additional multivariate analyses to assess the joint effects of a low/high Liano score, considering the median value $(0.22)$ as a cut-off, and the categories of RIFLE (no AKI or R vs category I or F), categorizing the participants in four (two $\mathrm{x}$ two) different groups. We combined RIFLE groups to maintain enough statistical power with the cross-classification categories because of the relatively small number of patients in our study.

In addition, we performed a joint score, combining both RIFLE categories and Liano score. We added to the Liano score the probability of death derived from the nonconditional logistic model for RIFLE I or F categories, using the following equation:

$\mathrm{P}=(\exp (-2.294+1.503 \times \mathrm{I}$ or F $)) /(1+\exp (-2.294+1.503 \times \mathrm{I}$ or F $))$

It was based on the fact that $\mathrm{P}=$ odds $/(1+$ odds $)$.

All P-values presented are two tailed; $\mathrm{P}<0.05$ was considered statistically significant. Statistical analyses were conducted using SPSS v.13.0 (SPSS Software, Chicago, IL, USA).

\section{RESULTS}

The four RIFLE groups differed in gender, admission type, proportion of oncology patients, pre-existing chronic renal failure, severity of illness (measured as Liano score), functional status at home (measured as Karnofsky score), prior food intake, causes of acute renal failure (ARF) and the further need for renal replacement therapy (Table 1). The mortality rate in the no AKI group was $4.2 \%$. In contrast, there was an increase from $20.5 \%$ in class $\mathrm{R}, 27.0 \%$ in class I to $33.4 \%$ in the class F.

The adjusted multivariate analyses (Table 2) showed that the RIFLE classes R, I and F were independent significant predictors of hospital mortality within 60 days. Patients in the risk (R) class had a HR of 2.77 (95\% CI, 1.15-6.66). Patients with injury (I) had a HR of 3.23 (95\% CI, 1.42-7.37), while those with failure (F) had a HR of $3.52(95 \%$ $\mathrm{CI}, 1.59-7.80)$. We recalculated $\mathrm{HR}$ with group $\mathrm{R}$ as the reference group, excluding those in the no AKI group. This examined whether R, I and F classification might be really useful in terms of predicting the risk. Unfortunately, neither group I nor F showed any significant adjusted HR for mortality, at 1.15 (95\% CI, 0.63-2.09) and 1.22 (95\% CI, 0.69-2.17), respectively.

We found additive effects of the exposures on in-hospital mortality from the crossclassification of the participants according to their Liano score values (median as a cutoff, 0.22) and RIFLE classes (Fig. 1). In other words, for any degree of AKI, hospital mortality increased as the Liano score increased. At the same time, patients with similar Liano scores but with more severe AKI had higher mortality rates. 
Cumulative survival rates within 60 days after starting the nephrology consultation differed significantly between groups (Fig. 2), consistent with our previous findings. The calibration curve shows that the proportion of patients who died increased in accordance with the increase in the severity of AKI. However, the result of the HosmerLemeshow goodness-of-fit test was statistically significant $(P=0.016)$, indicating a poor calibration ability (Fig. 3). That means that the predicted probabilities did not closely reflect the real risk.

The area under the ROC for the RIFLE criteria was smaller than the one for the Liano score, and the latter was smaller than the RIFLE criteria and Liano score combined (Fig. $4)$.

\section{DISCUSSION}

This study involved a cohort of 903 patients admitted to a tertiary-care academic medical centre and sought to examine the ability of the RIFLE criteria to predict inhospital mortality within 60 days.

We showed a significant increase of mortality associated with an increasing severity of AKI, as defined by the RIFLE criteria in this population. These findings are consistent with published reports ${ }^{19,20}$ and, more interestingly, the mortality rates were very similar to those recently reported by Bagshaw. ${ }^{21}$

Figure 2 shows a clear trend between RIFLE and the mortality. However, this trend is substantially reduced when adjusting for the potential confounding factors as shown in Table 2. We found that Liano score was the most important confounding factor. When adjusting for Liano score, it could explain the main magnitude reduction in the HR we obtained.

As several experts involved in the development of the RIFLE consensus have expressed their concerns about the misuse of the RIFLE criteria as a prognostic score system, ${ }^{14}$ our results should be interpreted with caution. The RIFLE criteria was not formulated to provide precise and robust prognosis information. AKI-specific predictive models, such as the Liano score, are more accurate for this purpose. Nonetheless, there is evidence showing an increase in mortality risk with a small increment in serum creatinine levels. $^{22}$

We calculated the cumulative survival rates according to recommendations for optimal follow-up time in patients with established ARF, ${ }^{9,23}$ Mortality related to other causes could bias any extended follow up.

With reference to the area under the ROC, the conventional threshold of 0.7 is considered satisfactory for clinical use. ${ }^{24}$ Logically, the Liano score proved to have a better discriminatory power than the RIFLE criteria. Comparing the RIFLE criteria with the Acute Physiology and Chronic Health Evaluation II (APACHE II) and Sequential Organ Failure Assessment (SOFA) scores, authors have found similar conclusions after analysing an intensive care unit population. ${ }^{25}$ However, a combined use of both RIFLE criteria and Liano score to perform a new model proved the best discriminatory power.

Finally, the RIFLE criteria showed poor calibration ability, thus slightly underestimating in-hospital mortality.

Our study also has some limitations. Urine output on a $6 \mathrm{~h}$ basis was not commonly measured in the wards, so we were unable to classify the patients according to this RIFLE criterion. Moreover, we started to collect the data before the whole definition was formulated. Besides, the exclusion of 44 oliguric patients without adequate serum creatinine increment might have changed the maximal classification. ${ }^{14}$ However, we 
believe that the influence of the misclassification of the diuresis variable would most likely have biased the HR toward the null value, reducing the magnitude of the risk estimates. The results remained statistically significant after adjusting for independent variables associated with mortality. A direct comparison of the predictive capacity of RIFLE criteria with and without the diuresis output might be needed to determine the importance of this value. ${ }^{12}$

Surprisingly, 44 patients from the original population presented oliguria but did not reach a minimum 50\% increase in their serum creatinine levels. Furthermore, only 267 patients $(28.6 \%)$ from the cohort were oliguric. The interpretation of this finding is difficult with the available data, thereby leaving us with no possible explanation.

However, we have minimized the influence of protein catabolism by grading the previous caloric consumption as well as adjusting for it in the analysis. Unfortunately, more accurate nutrition evaluation, such as serum prealbumin levels, was not measured in all patients.

Some authors might argue against resolving the MDRD equation to extrapolate the serum baseline creatinine values in our population. ${ }^{12,26}$ According to the advice of $\mathrm{ADQI}^{9}$ and the methods in other studies, ${ }^{6,7,20,21}$ we used the MDRD equation for baseline creatinine values: it may underestimate the severity of ARF when using the RIFLE criteria. The Liano score is not as well known as other prognostic scoring systems, although it might prove quite useful. Nevertheless, our findings should encourage future researchers to study other severity scoring systems.

Finally, because the study took place in a single hospital, our findings cannot be generalized to other centres.

One of the strengths of our study is that patients were further stratified using a severity of illness score before analysis. ${ }^{20,27} \mathrm{We}$ chose the Liano score because of its ability to discriminate mortality from survival and its ability to calibrate the observed mortality rate with the expected mortality in AKI patients. ${ }^{27-29} \mathrm{We}$ also took into account the functional capacity in the analysis, because we consider that this factor may be independently associated with mortality. ${ }^{30}$

We want to point out that the joint effects of the Liano score and the RIFLE classes augmented the predictive powers. Perhaps this finding is the most interesting in our study, because the calculation of one complemented the value of the other. They could be used together in future practice to arrive at a more accurate predictive model.

In conclusion, in this general hospital population, the risk of mortality during the AKI episode was positively associated with high RIFLE classes. We also showed that the RIFLE classification system had discriminative power in predicting hospital mortality within 60 days in AKI patients, but it was not better than a specific AKI predictive model. However, the combined use of both may give a more robust prognosis system that needs further investigation.

\section{ACKNOWLEDGEMENTS}

The authors are grateful to Dr Zubair Kabir for his constructive comments, and also to the editor 'YourProfessor' (http:// www.editavenue.com) for her assistance in editing this manuscript. 


\section{REFERENCES}

1. Waikar SS, Curhan GC, Wald R, McCarthy EP, Chertow GM. Declining mortality in patients with acute renal failure, 1988- 2002. J. Am. Soc. Nephrol. 2006; 17: 1143-50.

2. Xue JL, Daniels F, Star RA et al. Incidence and mortality of acute renal failure in Medicare beneficiaries, 1992-2001. J. Am. Soc. Nephrol. 2006; 17: 1135-42.

3. Waikar SS, Liu KD, Chertow GM. The incidence and prognostic significance of acute kidney injury. Curr. Opin. Nephrol. Hypertens. 2007; 16: 227-36.

4. Ympa YP, Sakr Y, Reinhart K, Vincent JL. Has mortality from acute renal failure decreased? A systematic review of the literature. Am. J. Med. 2005; 118: 827-32.

5. Herrera-Gutierrez ME, Seller-Perez G, Maynar-Moliner J, Sanchez-IzquierdoRiera JA. [Epidemiology of acute kidney failure in Spanish ICU. Multicenter prospective study FRAMI]. Med. Intensiva 2006; 30: 260-67. (In Spanish.)

6. Hoste EA, Clermont G, Kersten A et al. RIFLE criteria for acute kidney injury are associated with hospital mortality in critically ill patients: A cohort analysis. Crit. Care 2006; 10: R73.

7. Uchino S, Bellomo R, Goldsmith D, Bates S, Ronco C. An assessment of the RIFLE criteria for acute renal failure in hospitalized patients. Crit. Care Med. 2006; 34: 1913-17.

8. Uchino S, Kellum JA, Bellomo $\mathrm{R}$ et al. Acute renal failure in critically ill patients: A multinational, multicenter study. JAMA 2005; 294: 813-18.

9. Bellomo R, Ronco C, Kellum JA, Mehta RL, Palevsky P. Acute renal failure definition, outcome measures, animal models, fluid therapy and information technology needs: The Second International Consensus Conference of the Acute Dialysis Quality Initiative (ADQI) Group. Crit. Care 2004; 8: R204-12.

10. Kellum JA, Levin N, Bouman C, Lameire N. Developing a consensus classification system for acute renal failure. Curr. Opin. Crit. Care 2002; 8: 50914.

11. Maccariello E, Soares M, Valente C et al. RIFLE classification in patients with acute kidney injury in need of renal replacement therapy. Intensive Care Med. 2007; 33: 597-605.

12. Joannidis M. Classification of acute kidney injury: Are we there yet? Intensive Care Med. 2007; 33: 572-4.

13. Bellomo R, Kellum JA, Ronco C. Comment on 'RIFLE classification in patients with acute kidney injury in need of renal replacement therapy' by Maccariello et al. Intensive Care Med. 2007; 33: 1850.

14. Kellum JA, Bellomo R, Ronco C. Classification of acute kidney injury using RIFLE: What's the purpose? Crit. Care Med. 2007; 35: 1983-4.

15. Liano F, Gallego A, Pascual J et al. Prognosis of acute tubular necrosis: An extended prospectively contrasted study. Nephron 1993; 63: 21-31.

16. Liano G, Pascual J. Acute renal failure. Madrid Acute Renal Failure Study Group. Lancet 1996; 347 (8999): 479.

17. Karnofsky DA, Burchenal JH (eds). The Clinical Evaluation of Chemotherapeutic Agents in Cancer. New York: Columbia University Press, 1949. 
18. National Kidney Foundation. K/DOQI clinical practice guidelines for chronic kidney disease: Evaluation, classification, and stratification. Am. J. Kidney Dis. 2002; 39 (2 Suppl 1): S1-S266.

19. Bellomo R, Kellum JA, Ronco C. Defining and classifying acute renal failure: From advocacy to consensus and validation of the RIFLE criteria. Intensive Care Med. 2007; 33: 409-13.

20. Ostermann M, Chang RW. Acute kidney injury in the intensive care unit according to RIFLE. Crit. Care Med. 2007; 35: 1837- 43.

21. Bagshaw SM, George C, Dinu I, Bellomo R. A multi-centre evaluation of the rifle criteria for early acute kidney injury in critically ill patients. Nephrol. Dial. Transplant. 2008; 23: 1203-10.

22. Chertow GM, Burdick E, Honour M, Bonventre JV, Bates DW. Acute kidney injury, mortality, length of stay, and costs in hospitalized patients. J. Am. Soc. Nephrol. 2005; 16: 3365-70.

23. Bell M, Liljestam E, Granath F, Fryckstedt J, Ekbom A, Martling CR. Optimal follow-up time after continuous renal replacement therapy in actual renal failure patients stratified with the RIFLE criteria. Nephrol. Dial. Transplant. 2005; 20: 354- 60 .

24. Lemeshow S, Le Gall JR. Modeling the severity of illness of ICU patients. A systems update. JAMA 1994; 272: 1049-55.

25. Ahlstrom A, Kuitunen A, Peltonen $\mathrm{S}$ et al. Comparison of 2 acute renal failure severity scores to general scoring systems in the critically ill. Am. J. Kidney Dis. 2006; 48: 262-8.

26. Poggio ED, Nef PC, Wang $X$ et al. Performance of the CockcroftGault and modification of diet in renal disease equations in estimating GFR in ill hospitalized patients. Am. J. Kidney Dis. 2005; 46: 242-52.

27. Star RA. Treatment of acute renal failure. Kidney Int. 1998; 54: 1817-31.

28. Douma CE, Redekop WK, van der Meulen JH et al. Predicting mortality in intensive care patients with acute renal failure treated with dialysis. J. Am. Soc. Nephrol. 1997; 8: 111-17.

29. Uchino S, Bellomo R, Morimatsu $\mathrm{H}$ et al. External validation of severity scoring systems for acute renal failure using a multinational database. Crit. Care Med. 2005; 33: 1961-7.

30. Perez Valdivieso JR, Bes-Rastrollo M, Monedero P, De Irala J, Lavilla FJ. Karnofsky performance score in acute renal failure as a predictor of short-term survival. Nephrology (Carlton, Vic) 2007; 12: 533-8. 


\begin{tabular}{|c|c|c|c|c|c|}
\hline & $\begin{array}{l}\text { No AKI } \\
n=259\end{array}$ & $\begin{array}{c}\text { RIFLE R } \\
n=112\end{array}$ & $\begin{array}{c}\text { RIFLE I } \\
n=185\end{array}$ & $\begin{array}{c}\text { RIFLE F } \\
n=347\end{array}$ & $P$-value* \\
\hline Mortality (\%) & 4.2 & 20.5 & 27.0 & 33.4 & $<0.001$ \\
\hline Age, median years (IQR) & $62(19.75)$ & $60(20.25)$ & $62(18.00)$ & $63(18.00)$ & 0.70 \\
\hline Men (\%) & 72.8 & 73.7 & 69.1 & 69.1 & 0.24 \\
\hline Surgical (\%) & 14.8 & 27.9 & 23.5 & 24.3 & 0.02 \\
\hline Oncology patients $(\%)$ & 40.2 & 44.9 & 35.1 & 32.6 & 0.02 \\
\hline CRF (\%) & 65.9 & 26.3 & 12.0 & 28.0 & $<0.001$ \\
\hline Diabetic patients (\%) & 11.6 & 8.5 & 9.4 & 8.9 & 0.30 \\
\hline Karnofsky score, median (IQR) & $70(20)$ & $70(20)$ & $60(10)$ & $60(10)$ & $<0.001$ \\
\hline Liano scoring, median (IQR) & $0.16(0.13)$ & $0.19(0.17)$ & $0.25(0.40)$ & $0.30(0.38)$ & $<0.001$ \\
\hline Oliguria $(\%)$ & 0.0 & 16.1 & 34.6 & 52.0 & $<0.001$ \\
\hline Jaundice $(\%)$ & 4.3 & 13.6 & 27.2 & 30.6 & $<0.001$ \\
\hline Coma $(\%)$ & 1.1 & 5.9 & 14.1 & 18.0 & $<0.001$ \\
\hline Consciousness $(\%)$ & 90.9 & 85.6 & 76.4 & 77.4 & $<0.001$ \\
\hline Mechanical ventilation (\%) & 1.1 & 9.3 & 27.2 & 25.7 & $<0.001$ \\
\hline Nephrotoxicity (\%) & 42.4 & 42.4 & 42.9 & 43.1 & 0.84 \\
\hline Causes of AKI $(\%)$ & & & & & $<0.001$ \\
\hline Pre-renal & NA & 80.5 & 81.1 & 68.8 & \\
\hline Intrinsic renal & NA & 16.9 & 15.7 & 22.0 & \\
\hline Post-renal & NA & 1.7 & 1.6 & 3.7 & \\
\hline Other causes & NA & 0.8 & 1.6 & 5.4 & \\
\hline Need of RRT (\%) & 1.8 & 11.9 & 24.6 & 41.4 & $<0.001$ \\
\hline Food intake $(\%)$ & & & & & $<0.001$ \\
\hline Optimal nutrition & 60.1 & 39.8 & 33.3 & 25.1 & \\
\hline Mild malnutrition & 10.1 & 19.4 & 17.7 & 20.3 & \\
\hline Moderate malnutrition & 19.7 & 16.3 & 25.9 & 32.6 & \\
\hline Severe malnutrition & 10.1 & 24.5 & 23.1 & 22.0 & \\
\hline \multicolumn{6}{|c|}{$\begin{array}{l}* P \text {-value test with ANOVA test for continuous variables and Fisher's exact test for categorical } \\
\text { variables. AKI, acute kidney injury; CRF, chronic renal failure; F, failure; I, injury; IQR, interquartile } \\
\text { range; NA, not applicable; R, risk; RRT, renal replacement therapy. }\end{array}$} \\
\hline
\end{tabular}


Table 2. Incidence of in-hospital mortality, and hazard ratios and (95\% confidence intervals) for in-hospital mortality according to each RIFLE category in 903 patients.

\begin{tabular}{|l|c|c|c|c|c|}
\hline & $\begin{array}{c}\text { No AKI } \\
\boldsymbol{n}=\mathbf{2 5 9}\end{array}$ & $\begin{array}{c}\text { RIFLE R } \\
\boldsymbol{n}=\mathbf{1 1 2}\end{array}$ & $\begin{array}{c}\text { RIFLE I } \\
\boldsymbol{n}=\mathbf{1 8 5}\end{array}$ & $\begin{array}{c}\text { RIFLE F } \\
\boldsymbol{n}=\mathbf{3 4 7}\end{array}$ & $\begin{array}{c}\text { P for } \\
\text { trend }\end{array}$ \\
\hline $\begin{array}{l}\text { Incidence of in-hospital } \\
\text { mortality, } n(\%)\end{array}$ & $11(4.2)$ & $23(20.5)$ & $50(27.0)$ & $116(33.4)$ & $<0.001$ \\
\hline Crude HR (95\% CI) & 1.00 (Ref.) & $5.08(2.19-11.76)$ & $7.64(3.56-16.37)$ & $10.57(5.13-21.79)$ & $<0.001$ \\
\hline $\begin{array}{l}\text { Age- and sex-adjusted } \\
\text { HR (95\% CI) }\end{array}$ & 1.00 (Ref.) & $5.07(2.19-11.76)$ & $7.67(3.58-16.45)$ & $10.57(5.20-22.11)$ & $<0.001$ \\
\hline $\begin{array}{l}\text { Multivariate-adjusted } \\
\text { HR } \uparrow(95 \% \text { CI) }\end{array}$ & 1.00 (Ref.) & $2.77(1.15-6.66)$ & $3.23(1.42-7.37)$ & $3.52(1.59-7.80)$ & 0.005 \\
\hline
\end{tabular}

$\dagger$ Adjusted for age, sex, Liano scoring, prior food intake, need of renal replacement therapy, chronic renal failure, cause of AKI, Karnofsky score, oncologic disease and admission type (surgical or not). AKI, acute kidney injury; CI, confidence interval; F, failure; HR, hazard ratios; I, injury; R, risk. 


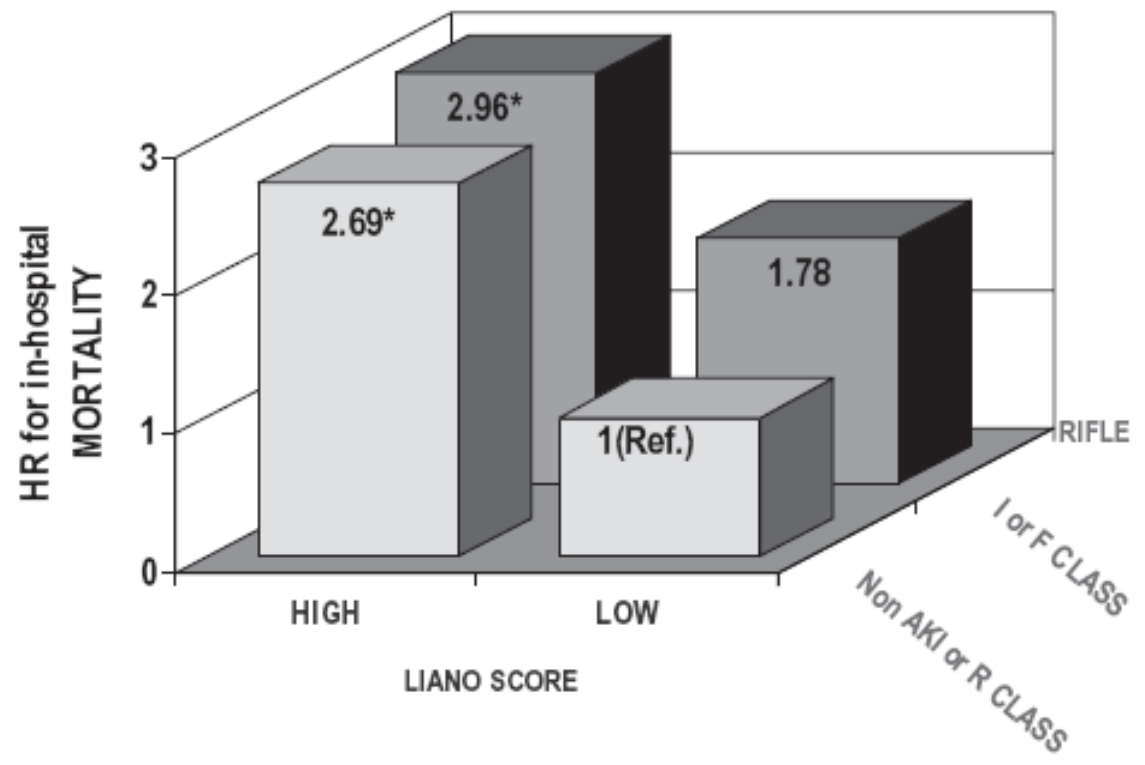

Figure 1. Adjusted hazard ratios (HR) for in-hospital mortality according to Liano score (LS) and risk, injury, failure, loss and end-stage renal failure (RIFLE) categories. HR were adjusted for age, sex, Liano score, prior food intake, need of renal replacement therapy, chronic renal failure, cause of acute kidney injury (AKI), Karnofsky score, oncologic disease and admission type (surgical or not). ${ }^{*} P<0.05$.

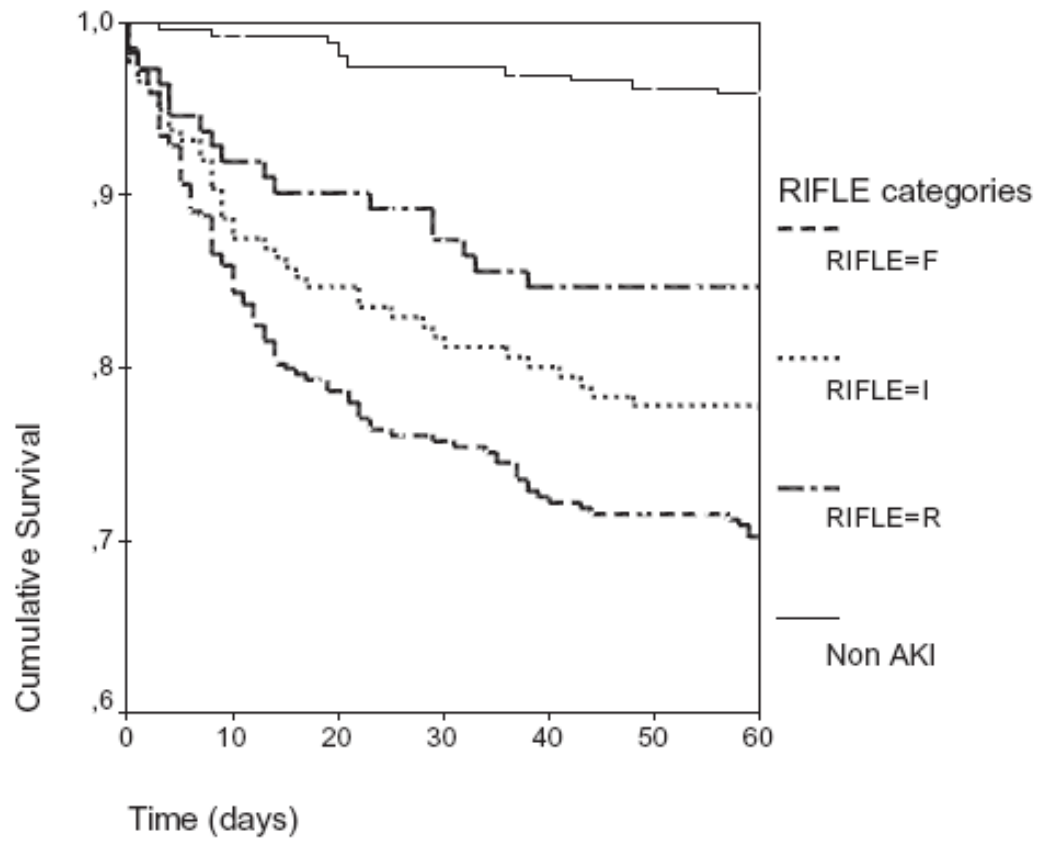

Figure 2. Sixty day survival after starting the nephrology consultation according to the RIFLE classes. Log-rank test, $P<0.01$. AKI, acute kidney injury; F, failure; I, injury; $\mathrm{R}$, risk. 


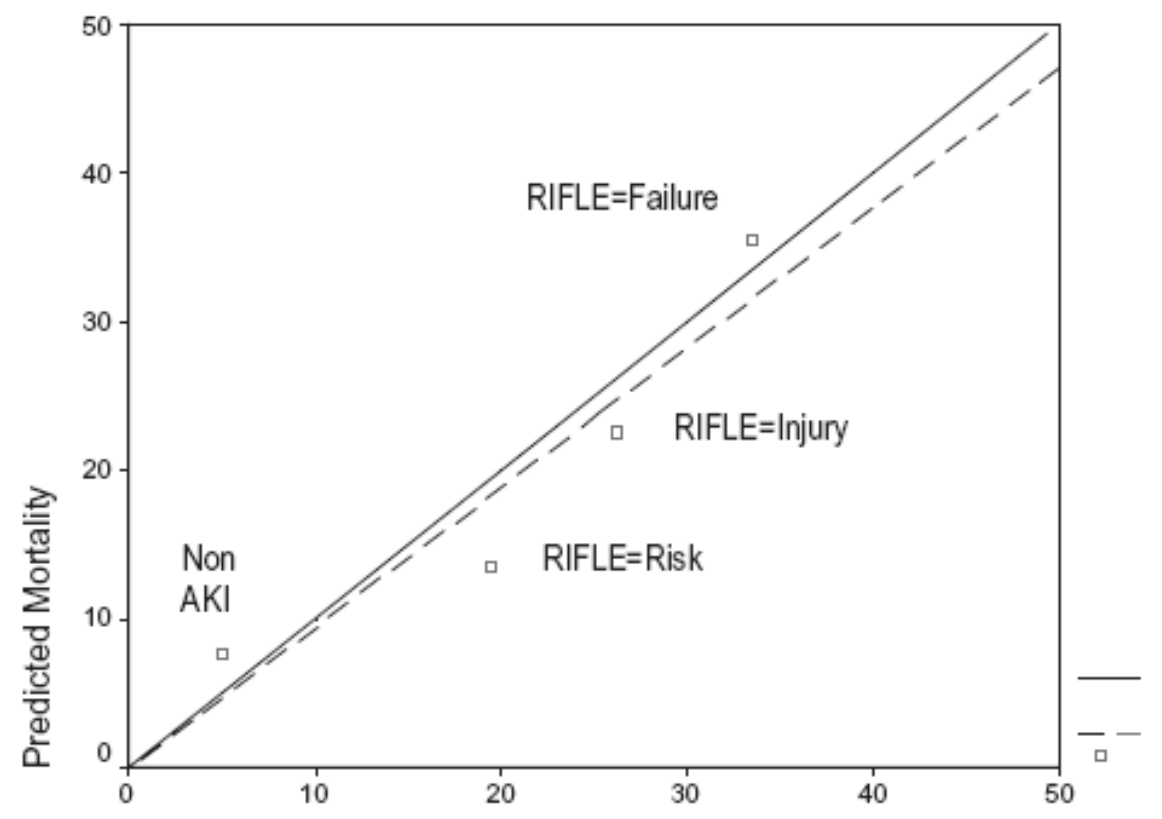

Observed Mortality

Figure 3. Calibration curves for RIFLE criteria. The continuous diagonal line is the line of ideal prediction (predicted mortality = observed mortality) for RIFLE criteria ( $\square$ ). Calibration curves below the diagonal line indicate that actual mortality was greater than the predicted. AKI, acute kidney injury; F, failure; I, injury; R, risk.



Figure 4. Receiver-operator curve (ROC) analysis for RIFLE criteria, Liano score, and RIFLE criteria and Liano score combined. Area under the ROC for RIFLE criteria: 0.69. Area under the ROC curve for Liano score: 0.78. Area under the ROC curve for RIFLE criteria and Liano score combined: 0.80 . 